\title{
VARIATIONAL PRINCIPLES IN ANISOTROPIC AND NONHOMOGENEOUS ELASTOKINETICS*
}

\author{
By M. BEN-AMOZ (General Electric Co.)
}

\begin{abstract}
Variational principles are formulated in terms of polarization stresses and strains in anisotropic and nonhomogeneous elastokinetics. The principles correspond to the well-known principles of Reissner and Washizu and are shown to produce, under suitable subsidiary conditions, functionals which are a generalization to elastokinetics of the Hashin-Shtrikman functionals in elastostatics.
\end{abstract}

1. Introduction. In recent years, new extremum principles have been discovered by Hashin-Shtrikman [1] in anisotropic and nonhomogeneous elastostatics, involving so-called polarization stresses and strains. According to this approach, the first and second boundary-value problems of elastostatics are rephrased in terms of a reference material and polarization stresses or strains. Variational principles are formulated in terms of the polarizations and an auxiliary field of either stress or strain deviations. The latter are obtained, following Hashin-Shtrikman, through exact solutions of subsidiary boundary-value problems involving the elastic moduli of the reference material. As was shown by Hill [2], these principles can be derived from the classical principles of minimum potential and complementary energy. The determination of the auxiliary fields often poses difficulties which are further enhanced in elastokinetics by the appearance of time as an additional independent variable in the subsidiary boundaryvalue problem.

In view of this it is desirable to formulate variation principles which allow a direct determination of both polarizations and deviations, in an approximate manner, from the principle. As the exact knowledge of the deviations is often not essential, the task of finding the auxiliary field is materially simplified by an independent approximation of polarizations and deviations within the functional. As is known, the theorems of Reissner [3] and Washizu [5] offer similar advantages as compared to the theorems of minimum potential or complementary energy.

In this paper, such principles are formulated ${ }^{* *}$ for both boundary-value problems of elastokinetics and it is shown that with suitable subsidiary conditions, additional functionals are produced which are generalizations of the Hashin-Shtrikman principles in elastostatics.

2. The elastokinetic equations. It will prove convenient, for present purposes, to recast the usual elastokinetic equations by introducing kinetic stress-strain relations and a kinetic measure of strain. This is achieved through a kinetic "stress" vector*** $\sigma_{i 4}$ and a kinetic "strain" vector $e_{i 4}$. Using boldface notation for tensors, the following equations are equivalent to the usual formulation. $\dagger$

$$
\nabla \cdot \boldsymbol{\sigma}=\dot{\sigma_{i 4}}
$$

*Received April 19, 1965.

**Analogous principles have been obtained in heat conduction and will be presented elsewhere.

***These are actually components of the material-energy tensor in relativity which are used here in a more restricted sense.

${ }^{\dagger} \boldsymbol{\nabla} \cdot \boldsymbol{\sigma}$ is used as a convenient form for $\sigma_{i j, j}$ in $(2.1)$. 


$$
\begin{aligned}
& \boldsymbol{\delta}=\mathbf{L} \cdot \mathbf{e}, \\
& \sigma_{i 4}=\rho \cdot e_{i 4}, \\
& \mathbf{e} \equiv e_{i j}=\frac{1}{2}\left(u_{i, i}+u_{i, i}\right) \quad(i=1, \cdots, 3 ; j=1, \cdots, 3) \text {, } \\
& e_{i 4}=u_{i}^{*} \text {. }
\end{aligned}
$$

Here $\boldsymbol{\delta}$ and $\mathbf{e}$ are the ordinary stress and strain tensors and $\mathbf{L}$ is the fourth rank tensor of elastic moduli with inverse $\mathbf{M}=\mathbf{L}^{-1}$. Equations (2.3) and (2.5) are, respectively, the kinetic stress-strain relations and strain measure, where the dot indicates time differentiation. Apart from any deeper significance (if any) the present formulation proves convenient in the derivation of variation principles in elastokinetics and in the transition from one principle to another.

3. Variational principle for prescribed surface displacements. We suppose that in a reference material with elastic moduli $\mathrm{L}^{0}$ and density $\rho^{0}$, the variables $\mathrm{o}^{0}, \mathrm{e}^{0}, \sigma_{i 4}^{0}, e_{i 4}^{\mathbf{0}}, u_{i}^{\mathbf{0}}$ are connected by relations (2.1)-(2.5). Furthermore, the displacements $u_{i}^{0}$ are supposed to be given on the boundary $S$. We consider next, a geometrically identical body of the actual material and define stress polarizations (elastic and kinetic) by the relations

$$
\begin{aligned}
\boldsymbol{\delta} & =\mathbf{L}^{0} \cdot \mathbf{e}+\tau, \\
\sigma_{i 4} & =\rho^{0} \cdot e_{i 4}+\mu_{i 4} .
\end{aligned}
$$

Correspondingly, elastic and kinetic strain deviations are given by

$$
\begin{aligned}
\mathrm{e}^{\prime} & =\mathrm{e}-\mathrm{e}^{0}, \\
e_{i 4}^{\prime} & =e_{i 4}-e_{i 4}^{0},
\end{aligned}
$$

which follows from the definition of displacement deviations

$$
u_{i}^{\prime}=u_{i}-u_{i}^{0} .
$$

The functional

$$
\begin{array}{rl}
2\left(J_{p}-J_{0}\right)=-\int_{t_{1}}^{t_{s}} d & d \int_{V}\left[\mathbf{e}^{\prime} \cdot \mathbf{L}^{0} \cdot \mathbf{e}^{\prime}-e_{i 4}^{\prime} \cdot \rho^{0} \cdot e_{i 4}^{\prime}+\tau\left(\mathbf{P} \cdot \tau-2 \mathbf{f}^{\prime}-2 \mathbf{e}^{0}\right)\right. \\
& \left.-\mu_{i 4}\left(R \cdot \mu_{i 4}-2 f_{i 4}^{\prime}-2 e_{i 4}^{0}\right)-2 \mathbf{f}^{\prime} \cdot \mathbf{L}^{0} \cdot \mathbf{e}^{\prime}+2 f_{i 4}^{\prime} \rho^{0} e_{i 4}^{\prime}\right] d V,
\end{array}
$$

where

$$
J_{0}=\frac{1}{2} \int_{t_{2}}^{t_{2}} d t \int_{V}\left[\mathbf{\sigma}^{0} \cdot \mathbf{e}^{0}-\boldsymbol{\sigma}_{i 4}^{0} \cdot e_{i 4}^{0}\right] d V
$$

and

$$
\begin{aligned}
\mathrm{f}^{\prime} & \equiv f_{i j}^{\prime}=\frac{1}{2}\left(u_{i, j}^{\prime}+u_{i, i}^{\prime}\right), \\
f_{i 4}^{\prime} & =u_{i}^{\prime}, \\
\mathrm{P} & =\left(\mathrm{L}-\mathrm{L}^{0}\right)^{-1}, \quad R=\left(\rho-\rho^{0}\right)^{-1},
\end{aligned}
$$

subject to the conditions

$$
u_{i}^{\prime}(S)=0
$$


on the surface $S$, and

$$
u_{i}^{\prime}\left(t_{1}\right)=u_{i}^{\prime}\left(t_{2}\right)=0
$$

at the extremities of the time interval, assumes a stationary value when

$$
\begin{aligned}
\nabla \cdot\left(\mathbf{L}^{0} \cdot \mathrm{e}^{\prime}+\tau\right) & =\left(\rho^{n} \cdot e_{i 4}^{\prime}+\mu_{i 4}\right), \\
\mathbf{P} \cdot \tau & =\mathrm{e}^{0}+\mathrm{f}^{\prime}, \\
R \cdot \mu_{i 4} & =e_{i 4}^{0}+f_{i 4}^{\prime}, \\
\mathrm{e}^{\prime}-\mathrm{f}^{\prime} & =0, \\
e_{i 4}^{\prime}-f_{i 4}^{\prime} & =0 .
\end{aligned}
$$

Integrating by parts and using (3.10), (3.11) and the divergence theorem, the first variation of (3.6) is

$$
\begin{aligned}
& 2 \delta J_{p}=-2 \int_{\mathbf{l}}^{t_{2}} d t \int_{V}\left[\mathbf{L}^{0} \cdot \delta \mathbf{e}^{\prime}\left(\mathbf{e}^{\prime}-\mathbf{f}^{\prime}\right)-\rho^{0} \cdot \delta e_{i_{1}}^{\prime}\left(c_{i_{4}}^{\prime}-f_{{ }_{4}}^{\prime}\right)+\delta \boldsymbol{z}\left(\mathbf{P} \cdot \tau-\mathbf{f}^{\prime}-\mathbf{e}^{\mathrm{n}}\right)\right. \\
& \left.\left.-\delta \mu_{i \pm}\left(R \cdot \mu_{i \neq}-f_{i 4}^{\prime}-e_{i \ddagger}^{0}\right)+\delta u_{i}^{\prime} \nabla \cdot\left(\mathbf{L}^{n} \cdot \mathbf{e}^{\prime}+\tau\right)-\left(\rho^{\prime \prime} e_{i \neq}^{\prime}+\mu_{i 4}\right)\right\}\right] d V .
\end{aligned}
$$

Consequently, $\delta J_{p}=0$ when (3.12)-(3.16) hold.

The functional (3.6) leads to all field equations and in this sense corresponds to Washizu's principle [5]. A more restricted principle follows if (3.15) and (3.16) are taken as subsidiary conditions in which case (3.6) reduces to

$2\left(J_{p}-J_{0}\right)=\int_{l_{1}}^{t_{2}} d t \int_{V}\left[\mathbf{e}^{\prime} \cdot \mathbf{L}^{n} \cdot \mathbf{e}^{\prime}-e_{i 4}^{\prime} \cdot \rho^{0} e_{i 4}^{\prime}-\tau(\mathbf{P} \cdot=-2 \mathbf{e})+\mu_{i 4}\left(R \cdot \mu_{i 4}-2 e_{i 4}\right)\right] d V$.

The functional (3.18) leads to (3.12)-(3.14) and thus corresponds to Reissner's principle [3]. While (3.6) is slightly more general, the desired simplification mentioned earlier in the introduction is effectively achieved through use of (3.18). If in addition to (3.15) and (3.16), (3.12) are taken as subsidiary conditions, (3.6) reduces to

$2\left(J_{p}-J_{0}\right)=-\int_{t_{1}}^{t_{2}} d t \int_{V}\left[\tau\left(\mathbf{P} \cdot \tau-\mathbf{e}^{\prime}-2 \mathbf{e}^{0}\right)-\mu_{i 4}\left(R \cdot \mu_{i 4}-e_{i 4}^{\prime}-2 e_{i t}^{0}\right)\right] d V$.

The functional (3.19), subject to the stated conditions, assumes a stationary value when (3.13) and (3.14) hold. This constitutes a generalization to elastokinetics of the Hashin-Shtrikman principle in elastostatics when surface displacements are prescribed [1].

Two limiting cases are of interest. When

$$
\mathrm{L}^{0} \rightarrow 0 \text { and } \rho^{0} \rightarrow 0
$$

(3.19) reduces, by the procedure of reference [1], to

$$
2 J_{p}=-\int_{t_{2}}^{t_{2}} d t\left[\int_{V}\left[\mathbf{\sigma} \cdot \mathbf{M} \cdot \mathbf{\sigma}-\sigma_{i t} \cdot 1 / \rho \cdot \sigma_{i t}\right] d V+\int_{S} \sigma_{i i} n_{i} u_{i} d S\right]
$$

while (3.12) reduces to

$$
\nabla \cdot \boldsymbol{d}=\sigma_{i 4}^{\cdot}
$$


The functional (3.20) subject to (3.21) is Hamilton's principle expressed in terms of stresses (elastic and kinetic) and is effectively equivalent to Castigliano's principle as extended by Reissner [4] to elastokinetics. On the other hand, when

$$
\mathrm{L}^{0} \rightarrow \infty \text { and } \rho^{0} \rightarrow \infty,
$$

again, by the procedure of reference [1], (3.19) reduces to Hamilton's principle

$$
2 J_{p}=\int_{t_{\mathbf{1}}}^{t_{\boldsymbol{q}}} d t \int_{V}\left[\mathrm{e} \cdot \mathrm{L} \cdot \mathrm{e}-e_{i 4} \cdot \rho \cdot e_{i 4}\right] d V
$$

expressed in terms of strains (elastic and kinetic) given by (2.4) and (2.5).

4. Variational principle for prescribed surface tractions. When surface tractions are prescribed, elastic and kinetic strain polarizations are defined:

$$
\begin{gathered}
\mathrm{e}=\mathbf{M}^{0} \cdot \boldsymbol{d}-\mathbf{n}, \\
e_{i 4}=\rho^{0-1} \cdot \sigma_{i 4}-\lambda_{i 4} .
\end{gathered}
$$

Accordingly, stress deviations (elastic and kinetic) are defined by

$$
\begin{aligned}
\boldsymbol{d}^{\prime} & =\boldsymbol{\delta}-\boldsymbol{\sigma}^{0}, \\
\sigma_{i 4}^{\prime} & =\sigma_{i 4}-\sigma_{i 4}^{0} .
\end{aligned}
$$

The functional

$$
\begin{aligned}
2\left(J_{p}-J_{0}\right)=-\int_{l_{2}}^{l_{2}} d t \int_{V}\left[\boldsymbol{\sigma}^{\prime} \cdot \mathbf{M}^{0} \cdot \boldsymbol{\sigma}^{\prime}-\sigma_{i 4}^{\prime} \cdot \rho^{0-1} \cdot \sigma_{i 4}^{\prime}+\mathbf{n}(\mathbf{Q} \cdot \mathbf{n}-2 \boldsymbol{\sigma})\right. \\
\left.-\lambda_{i 4}\left(S \cdot \lambda_{i 4}-2 \sigma_{i 4}\right)-2 \boldsymbol{d}^{\prime} \cdot \mathbf{f}+2 \sigma_{i 4}^{\prime} \cdot f_{i 4}\right] d V
\end{aligned}
$$

subject to the conditions

$$
\sigma_{i i}^{\prime}(S) n_{i}=0
$$

and

$$
v_{i}\left(t_{1}\right)=v_{i}\left(t_{2}\right)=0
$$

assumes a stationary value when

$$
\begin{aligned}
\nabla \cdot \mathbf{d}^{\prime} & =\sigma_{i 4}^{\prime \prime}, \\
\mathbf{Q} \cdot \mathbf{n} & =\mathbf{\sigma}, \\
S \cdot \lambda_{i 4} & =\sigma_{i 4}, \\
\mathbf{M}^{0} \cdot \mathbf{d}^{\prime}-\mathfrak{n} & =\mathbf{f}, \\
\rho^{0-\mathbf{1}} \cdot \sigma_{i 4}^{\prime}-\lambda_{i 4} & =f_{i 4},
\end{aligned}
$$

where

$$
\mathbf{Q}=\left(\mathbf{M}^{0}-\mathbf{M}\right)^{-1}, \quad S=\left(\rho^{0-1}-\rho^{-1}\right)^{-1}
$$

and

$$
\begin{aligned}
\hat{f} & \equiv f_{i i}=\frac{1}{2}\left(v_{i, i}+v_{i, i}\right), \\
f_{i 4} & =v_{i}^{\cdot}
\end{aligned}
$$


with $v_{i}$ as three arbitrary displacement functions. This corresponds to Washizu's principle [5] when surface tractions are prescribed over the entire surface. A more restricted principle corresponding to Reissner's is obtained if (4.11) and (4.12) are taken as subsidiary conditions. In this case (4.5) reduces to

$$
\begin{aligned}
2\left(J_{p}-J_{0}\right)=\int_{t_{1}}^{t_{2}} d t \int_{V}\left[\boldsymbol{\sigma}^{\prime} \cdot \mathbf{M}^{0} \cdot \boldsymbol{d}^{\prime}-\sigma_{i 4}^{\prime} \cdot \rho^{0-1} \cdot \sigma_{i 4}^{\prime}\right. \\
\\
\left.\quad-\mathbf{n} \cdot\left(\mathbf{Q} \cdot \mathbf{n}-2 \mathbf{\sigma}^{0}\right)+\lambda_{i 4}\left(S \cdot \lambda_{i 4}-2 \sigma_{i 4}^{0}\right)\right] d V
\end{aligned}
$$

which assumes a stationary value when (4.8)-(4.10) hold. If, in addition to (4.11) and (4.12), equations (4.8) are taken as subsidiary conditions, there follows from (4.5)

$2\left(J_{p}-J_{0}\right)=-\int_{t_{1}}^{t_{\mathbf{s}}} d t \int_{V}\left[\mathbf{n} \cdot\left(\mathbf{Q} \cdot \mathbf{n}-\mathbf{\sigma}^{\prime}-2 \mathbf{d}^{0}\right)-\lambda_{i 4}\left(S \cdot \lambda_{i 4}-\sigma_{i 4}^{\prime}-2 \sigma_{i 4}^{0}\right)\right] d V$

which assumes a stationary value when (4.9) and (4.10) hold. This represents a generalization to elastokinetics of the Hashin-Shtrikman principle [1] when surface tractions are prescribed. Again, it can be shown that in the limit as $\mathbf{M}^{0} \rightarrow 0$ and $\rho^{0-1} \rightarrow 0,(4.16)$ reduces to Hamilton's principle

$$
2 J_{p}=\int_{t_{2}}^{t_{\mathbf{s}}} d t \int_{V}\left[\mathbf{e} \cdot \mathbf{L} \cdot \mathbf{e}-e_{i 4} \cdot \rho \cdot e_{i 4}\right] d V
$$

whereas, when $\mathbf{M}^{0} \rightarrow \infty$ and $\rho^{0-1} \rightarrow \infty$ we obtain Hamilton's principle in terms of elastic and kinetic stresses.

\section{References}

1. Z. Hashin and S. Shtrikman, On some variational principles in anisotropic and nonhomogeneous elasticity, J. Mech. Phys. Solids, 10 (1962), pp. 335-342

2. R. Hill, New derivations of some elastic extremum principles, Progress in Appl. Mech., The Prager Anniversary Vol., Macmillan Co., 1963, p. 99

3. E. Reissner, On a variational theorem in elasticity, J. Math. Phys. 29 (1950), pp. 90-95

4. E. Reissner, On variational principles in elasticity, Proc. Symposia Appl. Math., vol.8, Mc Graw-Hill Book Co., New York, 1958, p. 1

5. K. Washizu, On the variational principles of elasticity and plasticity, Aeroelastic and Structure Res. Lab., MIT, 1955 